\title{
Spin Delocalization in the Radical Cations of Porphyrin Molecular Wires: A New Perspective on EPR Approaches
}

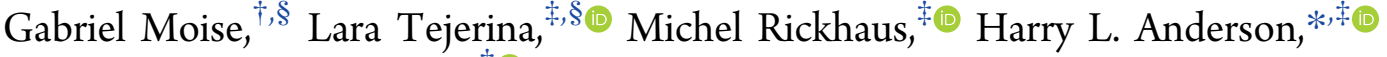 \\ and Christiane R. Timmel* ${ }^{\dagger} \dagger$ \\ ${ }^{\dagger}$ Centre for Advanced Electron Spin Resonance, Department of Chemistry, University of Oxford, Oxford OX1 3QR, United \\ Kingdom \\ ${ }^{\ddagger}$ Chemistry Research Laboratory, Department of Chemistry, University of Oxford, Oxford OX1 3TA, United Kingdom
}

Supporting Information

ABSTRACT: The spin delocalization in the radical cations of a series of ethynelinked oligoporphyrins was investigated using EPR spectroscopy. The roomtemperature spectral envelope for these oligomers deviates significantly from the benchmark $N^{-0.5}$ trend in line width expected for a completely delocalized spin density, in contrast to the butadiyne-linked analogues measured previously. Here, we show, using DFT calculations and complementary low-temperature ENDOR measurements, that this deviation is primarily driven by a more pronounced inequivalence of the ${ }^{14} \mathrm{~N}$ spins within individual subunits for the ethyne-linked oligoporphyrins. Once this ${ }^{14} \mathrm{~N}$ inequivalence is taken into consideration, the room-temperature and ENDOR spectra for both butadiyne-linked and ethynelinked oligomers, up to $N=5$, can be simulated by similar static delocalization patterns. This work highlights the importance of EPR in exploring such spin delocalization phenomena while also demonstrating that the $N^{-0.5}$ trend should not be interpreted in isolation but only in combination with careful simulation and theoretical modeling.
Non-uniform spin density

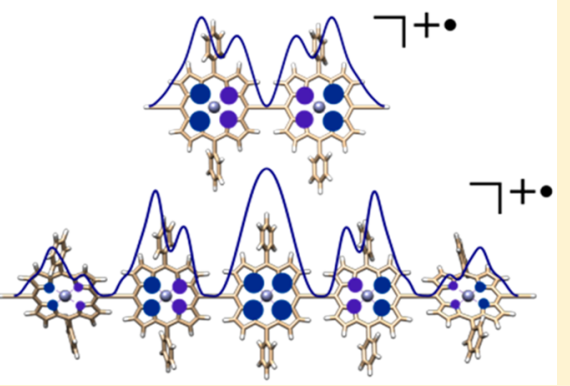

$\mathrm{M}$ olecular wires consisting of porphyrins linked by mesomeso ethyne bridges display long-range electronic communication resulting in strong near-infrared absorption and fluorescence, ${ }^{1}$ nonlinear optical behavior, ${ }^{2}$ and efficient charge transport. ${ }^{3}$ Here, we elucidate the extent of spin delocalization in these $\pi$-systems as a step toward understanding their behavior as organic semiconductors.

Electron paramagnetic resonance (EPR) has been used to investigate the spin delocalization in a wide range of paramagnetic porphyrin systems, such as radical cations and anions, $^{4-8}$ and photoexcited triplet states, ${ }^{9-12}$ of cyclic and linear oligomers. A previous study of the hole polaron states of ethyne-linked porphyrin oligomers indicated spin density delocalization lengths spanning up to seven porphyrin units (i.e., $>7.5 \mathrm{~nm}$ ). ${ }^{6}$ Following methodology originally introduced by Norris et al., ${ }^{13}$ these conclusions were based on narrowing of the line width of the continuous wave (cw) EPR signature of the radical cations with increasing oligomer length. In a recent study, ${ }^{4}$ room-temperature cw-EPR was combined with ${ }^{1} \mathrm{H}$ ENDOR of the frozen sample to fully characterize the spin delocalization in the radical cations of butadiyne-linked porphyrin oligomers. While at room temperature the cw-EPR line widths were observed to decrease as predicted for a fully delocalized spin density, at cryogenic temperatures, the spin was delocalized over only $2-3$ porphyrin units in linear and cyclic oligomers up to the hexamers. ${ }^{4}$

Here, we investigate the spin delocalization in radical cations of ethyne-linked porphyrin wires $\left(l-\mathbf{P N}^{+}\right.$, where $N=1-5,13$ porphyrin units) and a 6-porphyrin nanoring $\mathbf{c}$-P6 $\cdot \mathbf{T 6}^{+\bullet}$ (Figure 1). The oligomer $\mathbf{l - P 1 3}$ is the longest ethyne-linked porphyrin chain yet reported. The cyclic oligomer c-P6.T6 is included as a rigid system with reduced conformational disorder, in which all six sites are formally equivalent. ${ }^{14}$ Initially, the isotropic hyperfine couplings to ${ }^{14} \mathrm{~N}$ nuclei are obtained using room-temperature cw-EPR at the X-band $(\sim 9.4$ $\mathrm{GHz})$ before pulsed ${ }^{1} \mathrm{H}$-ENDOR at the Q-band $(\sim 34 \mathrm{GHz})$ at cryogenic temperatures is employed to probe the spin density more directly. DFT calculations yielding spin densities and hyperfine couplings support the experimental results.

Each oligomer is decorated with 1,3-bis(trihexylsilyl)phenyl substituents to enhance solubility in organic solvents and hinder aggregation. Terminal 3-cyanopropyldiisopropylsilyl (CPDIPS) protecting groups are chosen for the linear oligomers to facilitate purification. The monomer (l-P1), linear pentamer (l-P5), and cyclic hexamer c-P6·T6 were prepared as reported previously. ${ }^{14,15}$ Full synthetic protocols are given in the SI. The radical monocations $\mathbf{l}-\mathbf{P N}^{+\bullet}$ and $\mathbf{c}$-P6 • $\mathbf{T 6}^{+\bullet}$ were generated by oxidation with tris(4-bromophenyl)aminium hexachloroantimonate (BAHA) in a solvent mixture of toluene- $d_{8}:$ THF- $d_{8}: \mathrm{CD}_{2} \mathrm{Cl}_{2}$ (1:1:1 by volume), which forms a glass at low temperatures. ${ }^{4}$ Details of the oxidation protocol and EPR parameters are given in the SI.

Received: August 2, 2019

Accepted: August 28, 2019 

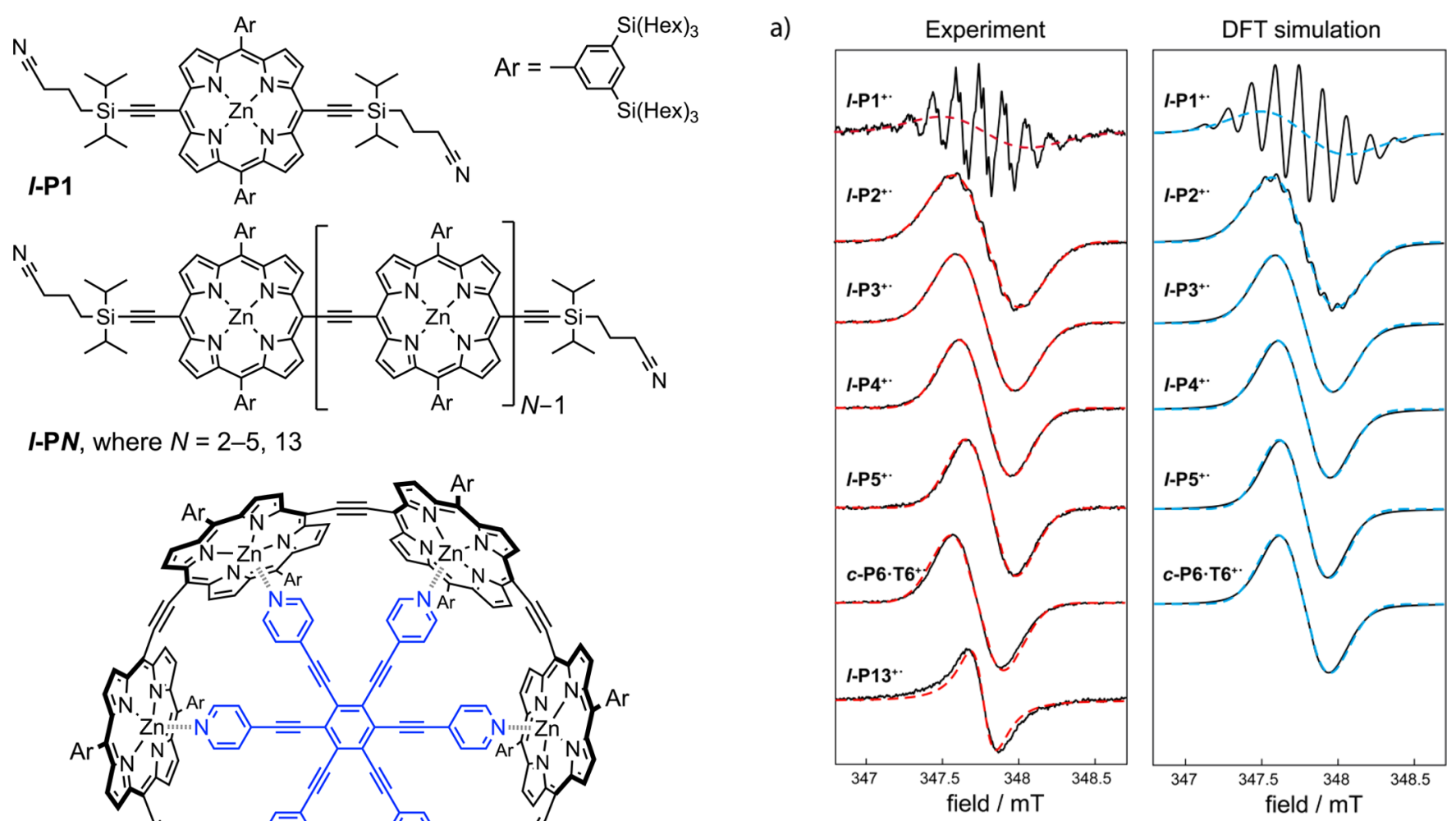

I-PN, where $N=2-5,13$

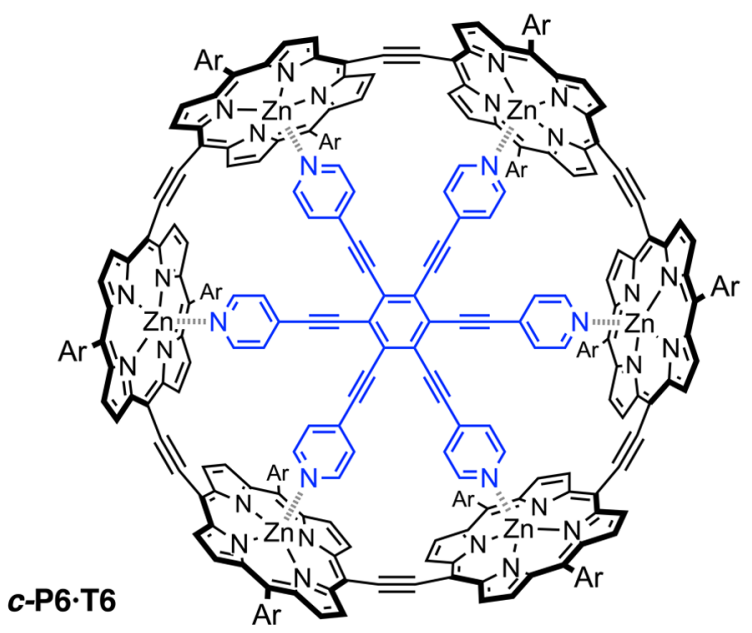

Figure 1. Structures of oligomers l-P1 to l-P13 and $c$-P6 6 T6.

The room-temperature X-band cw-EPR spectra of the oligomers $\mathbf{l}-\mathbf{P N}^{+\bullet}$ and $\mathbf{c}$-P6 $\mathbf{P} \mathbf{T}^{+\bullet}$ are depicted in Figures $2 \mathrm{a}$ (left) and 3 (left). The hyperfine structure in the spectrum of $l$ $\mathbf{P 1}^{+\bullet}$ is consistent with that reported previously for a similar monomer with equivalent acquisition parameters. ${ }^{4}$ As shown in Figure 3 (top and middle right panels), this hyperfine structure is primarily due to a coupling of $\sim 4.3 \mathrm{MHz}$ to the four equivalent ${ }^{14} \mathrm{~N}$ nuclei of the porphyrin, and it manifests as the larger peak-to-peak separation in the spectrum. In the $\mathbf{l - P 1 ^ { + \bullet }}$ spectrum, we also observe resolved superhyperfine structure (the smaller peak-to-peak separation), which can be modeled by four equivalent ${ }^{1} \mathrm{H}$ couplings of $\sim 1.0 \mathrm{MHz}$. These couplings were obtained from simulations using the EasySpin package (see the SI). ${ }^{16}$ The peaks in these spectra are also characterized by an inherent broadening, best simulated using a Lorentzian function, which for $\boldsymbol{l}_{\mathbf{P}} \mathbf{P} \mathbf{1}^{+\bullet}$ is parametrized by a line width, $\lambda_{1}=$ $0.059 \pm 0.016 \mathrm{mT}$, as seen in Figure 3 (bottom right). Because the inherent line width for the monomer is much smaller than the separation between the peaks, the overall spectral envelope (the square root of the variance of the spectrum) is dominated by the hyperfine couplings. Thus, provided the spin density is completely and uniformly delocalized (i.e., the ${ }^{14} \mathrm{~N}$ coupling in a particular oligomer, $A_{N}$, is inversely proportional to the number of porphyrin units, $\left.A_{N} \propto 1 / N\right)$, the relationship established by Norris (and discussed in the SI) should be applicable

$$
\Delta B_{N}=\frac{\Delta B_{1}}{\sqrt{N}}
$$

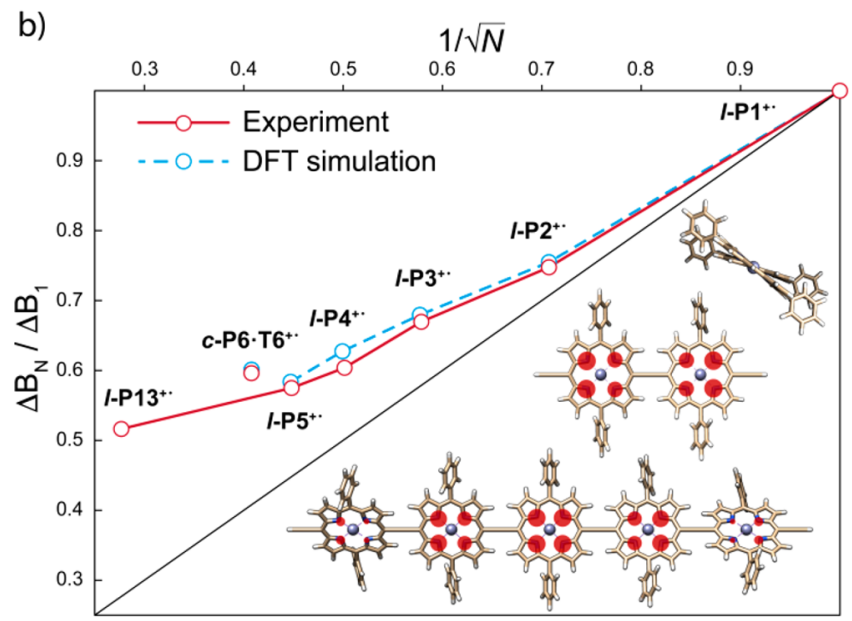

Figure 2. (a) (Left) Experimental X-band cw-EPR spectra of the radical cations $l$-P1 $1^{+\bullet}$ to $l-\mathrm{P} 3^{+\bullet}$ and $\mathrm{c}-\mathrm{P} 6 \cdot \mathbf{T 6}^{+\bullet}$ acquired at room temperature (black line) and the spectral envelope obtained by fitting a derivative Gaussian function (red dashed line). The spectral envelope of $\boldsymbol{l}-\mathrm{P} \mathbf{1} 3^{+\bullet}$ was fitted with a Lorentzian function (see the SI). (Right) EPR spectra obtained using the ${ }^{14} \mathrm{~N}$ spin densities from DFT calculations (black line). The best-fit derivative Gaussian envelope is plotted for each DFT spectrum (blue dashed line). (b) Normalized width of the experimental and DFT Gaussian envelopes vs $(1 / N)^{1 / 2}$. The inset shows the DFT optimized geometry of $l$-P2 ${ }^{+\bullet}$ (from two perspectives) and of $\mathbf{l}-\mathbf{P S}^{+\bullet}$ as well as the relative spin density on the nitrogen nuclei (see SI for more information).

where $\Delta B_{N}$ is the width of a Gaussian envelope fitted to the cw-EPR spectrum and $N$ is the number of porphyrin units. ${ }^{13}$ However, the experimental envelope line width obtained for this series of oligomers deviates from eq 1 by up to $\sim 25 \%$ (Figure 2b). One plausible explanation could be that the inherent line width, $\lambda_{N}$, of the peaks does not remain constant (or small) throughout the series. Initially, it might seem that there is a myriad of potential reasons for a change in $\lambda_{N}$ with 

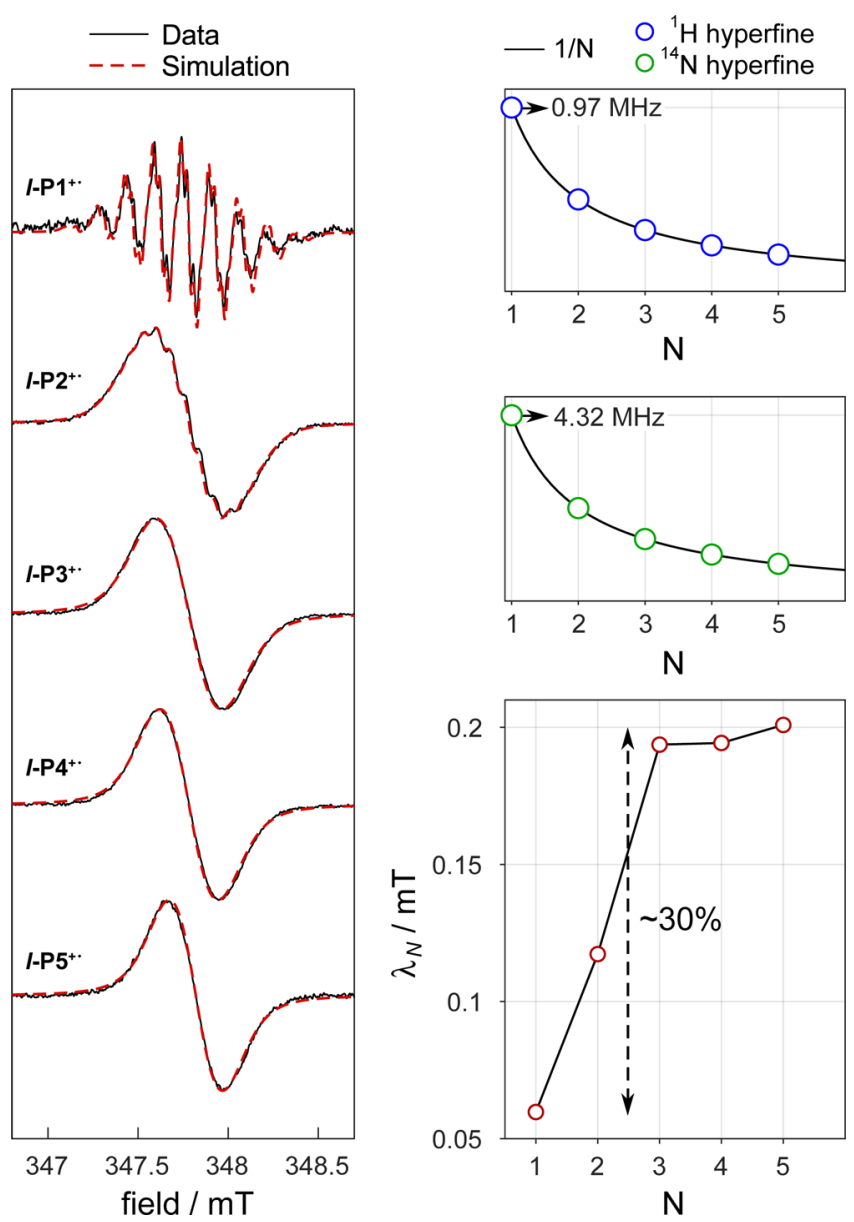

Figure 3. (Left) Numerical simulations (red dashed line) of the Xband cw-EPR spectra (black) of the linear radical cations up to $\mathbf{l}-\mathbf{P 5}^{+}$ $\left(\mathbf{l}-\mathrm{P}^{+} 3^{+}\right.$contains too many nuclei to allow simulations including both ${ }^{1} \mathrm{H}$ and ${ }^{14} \mathrm{~N}$ to be performed). (Right) Best-fit simulation parameters: ${ }^{1} \mathrm{H}$ hyperfine coupling (top), ${ }^{14} \mathrm{~N}$ hyperfine coupling (middle), and inherent line width (bottom). The best fit to the $\mathbf{l}_{-\mathbf{P 1}}{ }^{\bullet}{ }^{\bullet}$ spectrum was found using a three-parameter least-squares fitting of the ${ }^{1} \mathrm{H}$ and ${ }^{14} \mathrm{~N}$ couplings and an inherent Lorentzian line width, $\lambda_{1}$. The longer oligomers were fitted by dividing the $\mathbf{l}-\mathbf{P} \mathbf{1}^{+\bullet}$ couplings by $N$, increasing the number of nuclei by a factor of $N$, and allowing only the inherent line width to be varied in the fit.

increasing $N$, such as changes in rotational correlation and relaxation times, as well as different time scales for interporphyrin dihedral angle distortions. However, as shown in the top and middle right panels of Figure 3, if complete and uniform spin delocalization is imposed in the numerical simulation (i.e., $A_{N}=A_{1} / N$ ), the value of $\lambda_{N}$ required to fit the experimental spectra shows a significantly discontinuous trend (note the $\sim 30 \%$ increase in the bottom right panel of Figure $3)$. If this increase in $\lambda_{N}$ were, for example, due to a change in rotational correlation time as a function of molecular size, then we would expect $\lambda_{5}>\lambda_{4}>\lambda_{3}$ by analogy with the observed $\lambda_{3}>$ $\lambda_{2}>\lambda_{1}$. Moreover, considering the structural similarity between these oligomers and the butadiyne-linked counterparts investigated by Peeks, Tait, et al., ${ }^{4}$ for which $\lambda_{N}$ remains approximately constant and (crucially) approximately equal to $\lambda_{1}$, such a large change in inherent line width cannot be the reason for the trend in Figure $2 b$.

The only remaining assumption behind eq 1 is that the spin density is uniformly delocalized. ${ }^{13}$ To explore the possible breakdown of this assumption, the spin density of the oligomers was calculated using DFT (see the SI). A set of predicted EPR spectra was obtained (right panel in Figure 2a) by multiplying the Mulliken spin populations on the nitrogen atoms for the $N>1$ oligomers by the McConnell constant obtained from the spectrum of $\mathbf{l}-\mathbf{P 1}^{+\bullet}$ (see the SI) while the inherent line width was kept fixed. A derivative Gaussian envelope was then fitted to these DFT-predicted EPR spectra, yielding a trend in the envelope line width that closely follows the trend of the experimental spectra (Figure $2 b$ ).

Figure $2 \mathrm{~b}$ contains further crucial information on the longer oligomers: the spin densities in any noncentral porphyrin unit are up to $10 \%$ larger on the peripheral as compared to the central ${ }^{14} \mathrm{~N}$ spins, as highlighted for both $\boldsymbol{l}$-P2 ${ }^{+\bullet}$ and $\boldsymbol{l}$-P5 ${ }^{+\bullet}$ (see also Figure S17), providing a clue to the origin of the deviation from eq 1 . It follows that the spin density is distributed unevenly across the ${ }^{14} \mathrm{~N}$ nuclei of all porphyrin units in an oligomer. The DFT calculations indicate that this is due to both the inequivalence of the ${ }^{14} \mathrm{~N}$ spins in any single, noncentral porphyrin and the progressive depletion of spin density in more peripheral porphyrin subunits. Similar trends are observed in the butadiyne-linked oligomers, although, importantly, the inequivalence of central and peripheral ${ }^{14} \mathrm{~N}$ couplings within a porphyrin is less pronounced (Figures S17 and S18). ${ }^{4}$ Furthermore, it is now clear that it is possible to simulate the cw-EPR spectra reasonably well using the same inherent line width for both ethyne- and butadiyne-linked oligomers (Figure S19) if we no longer assume that all of the nuclei are equivalent.

In the analysis above, we have only considered the ${ }^{14} \mathrm{~N}$ spin densities for the DFT-optimized geometries, which for the dimers correspond to a $20^{\circ}$ dihedral angle in $\mathbf{l}-\mathbf{P 2} \mathbf{C}^{+\bullet}$ (Figure b) and approximately $0^{\circ}$ in the butadiyne-linked dimer. ${ }^{17-20}$ Although the energy barrier for rotation in both dimers exceeds $k T$ at room temperature, Figure S20 demonstrates that the butadiyne-linked dimer gives spectra that may be simulated equally well with either equivalent or thermally averaged but distinct central and peripheral ${ }^{14} \mathrm{~N}$ spins on each porphyrin unit, while the spectra obtained by these two methods differ significantly for $\mathbf{l}_{\mathbf{P 2}} \mathbf{P}^{+\bullet}$. The inequivalence of central and peripheral nitrogens in all but the monomer causes the breakdown of the Norris trend in these ethyne-linked oligomers.

The ${ }^{1} \mathrm{H}$ hyperfine couplings of the oligomers were obtained via pulse Mims-ENDOR experiments at $85 \mathrm{~K}$ and Q-band microwave frequencies. This EPR technique is a complementary method for determining hyperfine couplings not typically accessible in cw-EPR. While a direct and quantitative correlation between the room-temperature cw-EPR data and frozen solution measurements is not always possible, these pulse ENDOR measurements give us valuable insight into the electronic communication between the porphyrin units and, with accompanying spectral simulations, can validate the accuracy of the DFT calculations presented above. Dynamic polaron migration, or hopping, processes can sometimes occur rapidly on the time scale of the EPR experiment at room temperature and become slow at low temperature, leading to a change in the apparent spin density distribution. ${ }^{7}$ Another possible complication in the interpretation of the lowtemperature measurements is that the counterions (hexachloroantimonate) can adopt a fixed distribution of sites relative to the oligomer chain and thus cause distortions in the spin density by comparison to the room-temperature measure- 
ments. This issue was addressed in a previous study on the butadiyne-linked series whereby no major change was observed in the EPR spectra of the cations when either the identity of the counterion was varied or upon addition of high electrolyte concentrations aimed at screening the potential ion pairing effects. $^{4}$

The ${ }^{1} \mathrm{H}$-ENDOR spectra of these oligomers (Figure 4) are characteristic of protons in the weak coupling regime (i.e., the

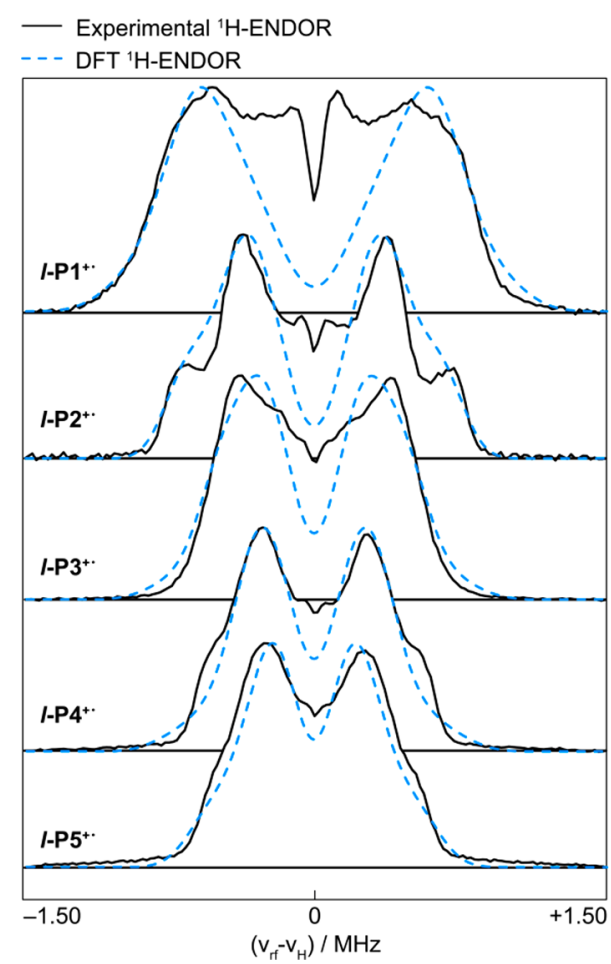

Figure 4. Q-band Mims ${ }^{1} \mathrm{H}$-ENDOR spectra of the radical cations $l$ $\mathbf{P 1}^{+\bullet}$ to $\mathbf{l}-\mathbf{P 5}^{+\bullet}$ acquired at $85 \mathrm{~K}$ together with simulated ${ }^{1} \mathrm{H}$-ENDOR spectra obtained from the DFT-calculated anisotropic hyperfine coupling tensors.

hyperfine couplings are much smaller than the proton Larmor frequency, $\nu_{\mathrm{H}} \approx 51 \mathrm{MHz}$ ). In this regime, the ${ }^{1} \mathrm{H}$-ENDOR spectra consist of symmetric peaks centered at the Larmor frequency and split by the hyperfine couplings.

A complete deconvolution of the ${ }^{1} \mathrm{H}$-ENDOR spectra into individual proton tensors (at least 14 protons need to be considered for the monomer alone) is neither practical nor necessary for our purposes. Instead, we present the experimental ${ }^{1} \mathrm{H}$-ENDOR spectra alongside those obtained using the DFT-computed hyperfine tensors as input to the function saffron in EasySpin (see the SI) and concentrate on a discussion of the most crucial changes observed within the series.

First, the simulated spectra qualitatively reproduce the appearance of the experimental spectra. Second, closer inspection of the series both in simulation and experiment reveals a decrease in spectral width by a factor of 1.6 from $l$ $\mathbf{P 1}^{+\bullet}$ to $\mathbf{l}-\mathbf{P S}^{+\bullet}$. This result is significant: if the spin density were uniformly delocalized over the porphyrin units, the width of the ${ }^{1} \mathrm{H}$-ENDOR spectra would be inversely proportional to $N$, by analogy with eq 1 .

Third, the ${ }^{1} \mathrm{H}$-ENDOR spectrum of $\mathbf{l}-\mathbf{P 2}^{+\bullet}$ contains two sets of distinctly resolved peaks (corresponding to hyperfine couplings of 0.7 and $1.4 \mathrm{MHz}$ ), whereas the butadiyne-linked counterpart spectrum was previously shown to contain only one set of resolved peaks (Figure S19c), consistent with a single average hyperfine coupling. ${ }^{4}$ Moreover, the ${ }^{1} \mathrm{H}$-ENDOR spectrum of the butadiyne-linked dimer has half of the width of the monomer spectrum, suggesting uniform spin delocalization. In contrast, the shoulders in the spectrum of $\mathbf{l}-\mathbf{P 2}^{+\bullet}$ indicate distortions in the spin density on the ${ }^{1} \mathrm{H}$ nuclei, thus not facilitating a simple delocalization argument based on the number of oligomer units. As shown in Figures S21 and S22, the proximity of the two porphyrin units causes the $\beta$-pyrrole protons close to the ethyne bridges to couple more strongly to the electron spin, leading to the observation of larger couplings in the ${ }^{1} \mathrm{H}$-ENDOR spectra, mirroring the inequivalence observed between the ${ }^{14} \mathrm{~N}$ couplings. The ENDOR spectrum of $\mathbf{l}-\mathrm{Pl}^{+\bullet}$ is essentially identical to that of $\mathbf{l}^{-\mathbf{P 5}^{+\bullet}}$ (Figure S21a), whereas the room-temperature spectrum of $\mathbf{l}-\mathbf{P 1 3}{ }^{+\bullet}$ is narrower than that of $\mathbf{l}-\mathbf{P 5}^{+\bullet}$ (Figure $2 \mathrm{a}$ ), which suggests that fast hopping may contribute toward the observed spin distribution in $l-\mathrm{P}^{+} 3^{+\bullet}$ at room temperature.

In summary, this work illustrates clearly that EPR is a powerful tool in investigating the spin delocalization in molecular wires when employed in combination with careful simulation and theory. As shown above, for the ethyne-linked oligomers investigated here, the assumptions behind eq 1 , typically employed to probe the extent of spin delocalization at room temperature within such wires, break down. The observation of a significant deviation from the $1 / \sqrt{N}$ trend could, at first sight, be easily interpreted as arising from reduced spin delocalization across the wire in the ethynelinked as compared to the butadiyne-linked structures, which follow this trend neatly. However, here we show the differences between the trends exhibited by the two oligomer series to be mainly due to an inhomogeneity in the ${ }^{14} \mathrm{~N}$ spin density within individual porphyrin units, which is more pronounced for the ethyne-linked wires. ${ }^{1} \mathrm{H}$-ENDOR further confirmed these distortions in spin density. Once this inhomogeneity in the ${ }^{14} \mathrm{~N}$ couplings is accounted for, the ethyne-linked oligomers exhibit a similar delocalization trend across the wire series as compared to the butadiyne analogues. This result therefore highlights that any conclusion on trends in spin density cannot be solely based on an interpretation of cw-EPR using eq 1 but must be accompanied by thorough computational analysis and, if possible, further experimental data, such as ENDOR spectra.

\section{ASSOCIATED CONTENT}

\section{S Supporting Information}

The Supporting Information is available free of charge on the ACS Publications website at DOI: 10.1021/acs.jpclett.9b02262.

Compound synthesis and characterization, experimental methods, cw-EPR and ENDOR simulations, and DFT calculations (PDF)

DFT Cartesian coordinates and spin densities (ZIP)

\section{AUTHOR INFORMATION}

\section{Corresponding Authors}

*E-mail: harry.anderson@chem.ox.ac.uk (H.L.A.).

*E-mail: christiane.timmel@chem.ox.ac.uk (C.R.T.).

ORCID $\odot$

Lara Tejerina: 0000-0003-1000-6310

Michel Rickhaus: 0000-0002-6107-2310 
Harry L. Anderson: 0000-0002-1801-8132

Christiane R. Timmel: 0000-0003-1828-7700

\section{Author Contributions}

${ }^{\S}$ G.M. and L.T. contributed equally to this work.

\section{Notes}

The authors declare no competing financial interest.

\section{ACKNOWLEDGMENTS}

We thank the EPSRC (EPL011972/1), the Swiss National Science Foundation (P2BSP2 168919), the ERC (320969), the SCG Innovation Fund, and the EC H2020 SYNCHRONICS (643238) for funding, the EPSRC UK National Mass Spectrometry Facility at Swansea University for MALDI spectra, and the University of Oxford Advanced Research Computing Service (ARC) for support. We acknowledge Prof. Michael J. Therien from Duke University in Durham, North Carolina, USA for insightful discussions about this work. We thank Dr. Claudia Tait from Freie Universität, Berlin, Germany for her comments, suggestions, and discussions about the EPR measurements, simulations, and DFT calculations presented here. G.M. acknowledges the financial support of the 1379 Society Old Members Scholarship from New College, University of Oxford.

\section{REFERENCES}

(1) (a) Bai, Y.; Olivier, J.-H.; Yoo, H.; Polizzi, N. F.; Park, J.; Rawson, J.; Therien, M. J. Molecular Road Map to Tuning Ground State Absorption and Excited State Dynamics of Long-Wavelength Absorbers. J. Am. Chem. Soc. 2017, 139, 16946. (b) Duncan, T. V.; Susumu, K.; Sinks, L. E.; Therien, M. J. Exceptional Near-Infrared Fluorescence Quantum Yields and Excited-State Absorptivity of Highly Conjugated Porphyrin Arrays. J. Am. Chem. Soc. 2006, 128, 9000. (c) Ostrowski, J. C.; Susumu, K.; Robinson, M. R.; Therien, M. J.; Bazan, G. C. Near-Infrared Electroluminescent Light-Emitting Devices Based on Ethyne-Bridged Porphyrin Fluorophores. Adv. Mater. 2003, 15, 1296. (d) Lin, V. S.-Y.; DiMagno, S. G.; Therien, M. J. Highly conjugated, acetylenyl bridged porphyrins: new models for light-harvesting antenna systems. Science 1994, 264, 1105.

(2) (a) Park, J. K.; Fischer, M. C.; Susumu, K.; Therien, M. J.; Warren, S. W. Femtosecond pulse train shaping improves two-photon excited fluorescence measurements. Opt. Lett. 2014, 39, 5606. (b) Drobizhev, M.; Stepanenko, Y.; Dzenis, Y.; Karotki, A.; Rebane, A.; Taylor, P. N.; Anderson, H. L. Extremely Strong Near-IR TwoPhoton Absorption in Conjugated Porphyrin Dimers: Quantitative Description with Three-Essential-States Model. J. Phys. Chem. B 2005, 109, 7223.

(3) (a) Bruce, R. C.; Wang, R.; Rawson, J.; Therien, M. J.; You, W. Valence Band Dependent Charge Transport in Bulk Molecular Electronic Devices Incorporating Highly Conjugated Multi-[(Porphinato) Metal] Oligomers. J. Am. Chem. Soc. 2016, 138, 2078. (b) Li, Z.; Park, T.-H.; Rawson, J.; Therien, M. J.; Borguet, E. Quasi-Ohmic Single Molecule Charge Transport through Highly Conjugated mesoto-meso Ethyne-Bridged Porphyrin Wires. Nano Lett. 2012, 12, 2722.

(4) Peeks, M. D.; Tait, C. E.; Neuhaus, P.; Fischer, G. M.; Hoffmann, M.; Haver, R.; Cnossen, A.; Harmer, J. R.; Timmel, C. R.; Anderson, H. L. Electronic Delocalization in the Radical Cations of Porphyrin Oligomer Molecular Wires. J. Am. Chem. Soc. 2017, 139, 10461 .

(5) Rawson, J.; Angiolillo, P. J.; Therien, M. J. Extreme electron polaron spatial delocalization in $\pi$-conjugated materials. Proc. Natl. Acad. Sci. U. S. A. 2015, 112, 13779.

(6) Susumu, K.; Frail, P. R.; Angiolillo, P. J.; Therien, M. J. Conjugated Chromophore Arrays with Unusually Large Hole Polaron Delocalization Lengths. J. Am. Chem. Soc. 2006, 128, 8380.

(7) Wilson, T. M.; Hori, T.; Yoon, M.-C.; Aratani, N.; Osuka, A.; Kim, D.; Wasielewski, M. R. Rapid Intramolecular Hole Hopping in meso-meso and meta-Phenylene Linked Linear and Cyclic Multiporphyrin Arrays. J. Am. Chem. Soc. 2010, 132, 1383.

(8) Kelley, R. F.; Tauber, M. T.; Wasielewski, M. R. LinkerControlled Energy and Charge Transfer within Chlorophyll Trefoils. Angew. Chem., Int. Ed. 2006, 45, 7979.

(9) Richert, S.; Limburg, B.; Anderson, H. L.; Timmel, C. R. On the Influence of the Bridge on Triplet State Delocalization in Linear Porphyrin Oligomers. J. Am. Chem. Soc. 2017, 139, 12003.

(10) Richert, S.; Bullard, G.; Rawson, J.; Angiolillo, P. J.; Therien, M. J.; Timmel, C. R. On the Importance of Electronic Symmetry for Triplet State Delocalization. J. Am. Chem. Soc. 2017, 139, 5301.

(11) Tait, C. E.; Neuhaus, P.; Peeks, M. D.; Anderson, H. L.; Timmel, C. R. Transient EPR Reveals Triplet State Delocalization in a Series of Cyclic and Linear $\pi$-Conjugated Porphyrin Oligomers. J. Am. Chem. Soc. 2015, 137, 8284.

(12) Angiolillo, P. J.; Rawson, J.; Frail, P. R.; Therien, M. J. The evolution of spin distribution in the photoexcited triplet state of ethyne-elaborated porphyrins. Chem. Commun. 2013, 49, 9722.

(13) Norris, J. R.; Uphaus, R. A.; Crespi, H. L.; Katz, J. J. Electron spin resonance of chlorophyll and the origin of signal $\mathrm{I}$ in photosynthesis. Proc. Natl. Acad. Sci. U. S. A. 1971, 68, 625.

(14) Rickhaus, M.; Vargas Jentzsch, A.; Tejerina, L.; Grübner, I.; Jirasek, M.; Claridge, T. D. W.; Anderson, H. L. Single-Acetylene Linked Porphyrin Nanorings. J. Am. Chem. Soc. 2017, 139, 16502.

(15) Haver, R.; Tejerina, L.; Jiang, H.-W.; Rickhaus, M.; Jirasek, M.; Grübner, I.; Eggimann, H. J.; Herz, L. M.; Anderson, H. L. Tuning the Circumference of Six-Porphyrin Nanorings. J. Am. Chem. Soc. 2019, $141,7965$.

(16) Stoll, S.; Schweiger, A. EasySpin, a comprehensive software package for spectral simulation and analysis in EPR. J. Magn. Reson. 2006, 178,42 .

(17) Lin, V. S.-Y.; Therien, M. J. The Role of Porphyrin-toPorphyrin Linkage Topology in the Extensive Modulation of the Absorptive and Emissive Properties of a Series of Ethynyl- and Butadiynyl-Bridged Bis- and Tris(porphinato)zinc Chromophores. Chem. - Eur. J. 1995, 1, 645.

(18) Peeks, M. D.; Neuhaus, P.; Anderson, H. L. Experimental and computational evaluation of the barrier to torsional rotation in a butadiyne-linked porphyrin dimer. Phys. Chem. Chem. Phys. 2016, 18, 5264.

(19) Winters, M. U.; Kärnbratt, J.; Eng, M.; Wilson, C. J.; Anderson, H. L.; Albinsson, B. Photophysics of a Butadiyne-Linked Porphyrin Dimer: Influence of Conformational Flexibility in the Ground and First Singlet Excited State. J. Phys. Chem. C 2007, 111, 7192.

(20) Rintoul, L.; Harper, S. R.; Arnold, D. P. A systematic theoretical study of the electronic structures of porphyrin dimers: DFT and TDDFT calculations on diporphyrins linked by ethane, ethene, ethyne, imine, and azo bridges. Phys. Chem. Chem. Phys. 2013, 15, 18951. 